\title{
FaceMemNet: Predicting Face Memorability with Deep Neural Networks
}

\author{
Mohammad Younesi ${ }^{1,2}$ and Yalda Mohsenzadeh ${ }^{1,2}$ \\ ${ }^{1}$ Department of Computer Science, Western University, London, Ontario, Canada \\ ${ }^{2}$ The Brain and Mind Institute, Western University, London, Ontario, Canada
}

\begin{abstract}
With the advent of social media in our day to day life, we are exposed to plenty of images, especially face photographs, every day. Recent behavioural studies have shown that some of these photographs stick in the mind better than others. However, it is still unclear what factors make a picture more or less memorable. Previous research have shown that memorability is an intrinsic property of an image, hence the memorability of an image can be computed from that image. Moreover, various works found that the memorability of an image is highly consistent across people and also over time. Recently, researchers employed deep neural networks to predict image memorability. Here, we show although those models perform well on scene and object images, they perform poorly on photographs of human faces. We demonstrate and explain why generic memorability models do not result in an acceptable performance on face photographs and propose seven different models to estimate the memorability of face images. In addition, we show that these models outperform the previous classical methods, which were used for predicting face memorability.
\end{abstract}

Every day, we meet new people or encounter new faces on social media. Some of these faces stick in our minds, while others are forgotten quickly. Despite individual differences in remembering visual events ${ }^{1,2}$, it has been shown that the image's memorability is consistent across people and over various time lags ${ }^{3-10}$. In other words, people show a consistent behaviour in remembering some images and forgetting other images. These findings raise the idea of estimating the memorability of an image, only from that image, i.e. predicting what images are more or less memorable than others ${ }^{11}$.

Previous research has shown that people fail to predict which images are more or less memorable than others 5 . Various research have explored the effect of context and image distinctiveness on memorability. It was shown that distinctive faces are recognized better than typical faces ${ }^{12}$. Another work has demonstrated that people tend to memorize faces rated as unusual in appearance better than typical faces ${ }^{13-15}$. Bainbridge et al. ${ }^{3}$ investigated what components are contributing to face memorability. They examined the role of twenty personalities (e.g. interesting/boring and calm/aggressive), social, and memory-related traits that have an impact on face memorability. After running a multiple linear regression model on these face attributes and memorability scores, they found that the combination of these attributes can only explain a small portion of the variance of the memorability scores. This suggests that the memorability of an image, lies on the image itself and it's hard to find the memorability of the image through some limited meaningful attributes.

Other works aimed to predict image memorability. Khosla et al. ${ }^{16}$ proposed one of the earliest methods using dense global features such as $\mathrm{HOG}^{17}$ and SIFT ${ }^{18}$ for predicting face memorability. The problem with these methods is that they are not fully automatic and they need to be tuned by a person. Convolutional neural networks ${ }^{19}$ have shown great performance in image classification $\operatorname{task}^{20}$ and since then have been used in various computer vision and machine learning tasks. Khosla et al. ${ }^{11}$ introduced the first model that used a convolutional neural network model called MemNet for predicting image memorability. It was trained by fine-tuning Hybrid-CNN ${ }^{21}$ and performed near human consistency rank correlation. Most recent works have employed attention models ${ }^{22}$ and residual networks ${ }^{23}$ to improve the model's performance in predicting memorability. Lu et al. ${ }^{24}$ tried to find out what are the elements that make outdoor natural scenes memorable. They discovered combining high-level features of scene category and deep features can result in improving the model performance in predicting memorability. While memorability is an intrinsic feature of an image, some works studied the extrinsic effects such as eye movements in predicting the memorability 25 .

We see people's faces in different conditions e.g. while they are happy, angry, or neutral. Bainbridge et al. ${ }^{26}$ shed light on how memorability changes with different transformations of the human face (neutral, happy, angry, 3/4 view, and profile view). They found that memorability is highly consistent within each image transformation as well. It means regardless of the person's face being neutral or happy, if she has got a memorable face, we'll remember her face and vice versa.

In this work, we have focused on predicting the memorability of faces. As Squalli-Houssaini et al. ${ }^{27}$ have demonstrated, deep neural network models (including MemNet $^{11}$ and other memorability networks that are trained on LaMem data set) succeed in predicting the memorability of scene and objects images. However, we show that the memorability models (including MemNet) that are trained on LaMem do not perform well in predicting memorability of face images. Here, using 10k US 
Adult Faces Database ${ }^{3}$, we fine-tuned VGG16 ${ }^{28}$, ResNet50 $0^{29}$ and SENet50 $0^{30}$ which are pre-trained on VGGFace2 data set ${ }^{31}$ to predict the face memorability scores. We also fine-tuned MemNet which is trained and performs well on LaMem ${ }^{11}$ data set to predict face memorability. Our results show that the fine-tuned models that are pre-trained on faces outperform the models which are pre-trained on LaMem. Our proposed models outperformed the previous model ${ }^{16}$ and got close to human consistency correlation in predicting face memorability.

\section{Results}

Deep neural network methods for predicting image memorability have been proposed previously ${ }^{11,22,23}$. MemNet ${ }^{11}$ is the first and most popular memorability predicting convolutional neural network trained on a large scale image memorability dataset $\left(\mathrm{LaMem}^{11}\right)$. LaMem is the largest annotated image memorability dataset available to date, consisting of $45 \mathrm{k}$ train images, $4 \mathrm{k}$ validation, and $10 \mathrm{k}$ test samples. MemNet could reach 0.64 rank correlation performance with the ground truth memorability scores of the held-out test set in LaMem. This data-set mainly contains scene-centric and object-centric images. While there are images including people in this dataset, this dataset was not designed nor included face images. Therefore, we hypothesized that models trained on this dataset will not perform well in predicting face memorability. In the next section, we first tested this hypothesis by evaluating the state-of-the-art memorability predicting deep neural networks on face memorability prediction.

\section{State-of-the-art memorability models fail to predict face memorability}

We evaluated three state-of-the-art memorability models which are trained on LaMem dataset on the task of predicting face memorability. For this, we used 10k US Adult Faces Database ${ }^{3}$ which contains 10,168 natural face photographs. 2222 of these faces were annotated with memorability scores.All the pictures in this dataset are oval-shaped with the same height and width.

We tested MemNet on the task of predicting memorability scores of the 10k US Adult Faces Database. Figure 1 depicts the architecture of the MemNet. The original MemNet is an old version and only available on Caffe. Therefore, we followed the methods described in the paper and retrained the model in PyTorch ${ }^{32}$. MemNet leverages AlexNet ${ }^{20}$ as its backbone. AlexNet showed a great performance in image classification task in 2012 and it began a revolution in this computer vision task. MemNet was the first model that utilized deep convolutional neural network for predicting image memorability and it outperformed all of its previous models that were designed to predict memorability scores. MemNet achieves $\mathbf{0 . 6 4}$ rank correlation on memorability scores corrected for false alarms and $\mathbf{0 . 5 7}$ on pure hit rate memorability scores in LaMem dataset. The architecture of MemNet consists of five convolutional and three max-pooling blocks that extract the features for predicting image memorability. Our Pytorch MemNet model reached the same level of performance on LaMem dataset that was mentioned in the original paper ${ }^{11}$. In addition to MemNet, we also fine-tuned two other models; (MemVGG depicted in Figure 2, and IncResMem depicted in Figure 3) using LaMem dataset to test them on the 10k US Adult Faces Database. MemVGG is the memorability predicting model with VGG16 as the backbone architecture. VGG16 is a newer model in comparison to AlexNet and achieves $92.7 \%$ top-5 test accuracy on the ImageNet dataset classification while AlexNet achieves $84.6 \%$ top-5 test accuracy. This model consists of fifteen convolutional blocks to extract the features followed by three fully connected layers to find the output (image classification on ImageNet ${ }^{33}$ or image memorability on LaMem). We fine-tuned a hybrid VGG16 model which was pre-trained on Places ${ }^{34}$ and ImageNet dataset to predict memorability scores on LaMem dataset. The average performance of this model was $\mathbf{0 . 6 3}$ on 5 test splits of LaMem dataset, however it does not require any complicated preprocessing steps like original AlexNet-based MemNet (see Methods section for more detail). The third model we trained on LaMem dataset is IncResMem. Recently Inception models have shown an outstanding performance in different machine vision tasks like image classification. Moreover, it has been shown that training with residual connections accelerates the training of Inception networks significantly. Therefore, we decided to use InceptionResNet ${ }^{35}$ for image memorability prediction. One of the benefit of InceptionResNetv2 model is that it is impervious to noisy labels. Another important feature of residual networks is that they can have very deep architecture while avoiding vanishing gradient problem. We took a pre-trained InceptionResNetv 2 model from Keras ${ }^{36}$ that was pre-trained on ImageNet for image classification. This model is deeper in comparison with the previous two models. To obtain better performance, we combined the categories from InceptionResNetV2 with VGG16 features to build the IncResMem model (see Figure 3). In other words, this model uses both semantic features of the images and also images categories. Stem, Inception and Reduction blocks are identical to the blocks that were introduced by Szegedy et al. ${ }^{35}$. This model achieved $\mathbf{0 . 6 4 6}$ rank correlation score on the LaMem dataset. 


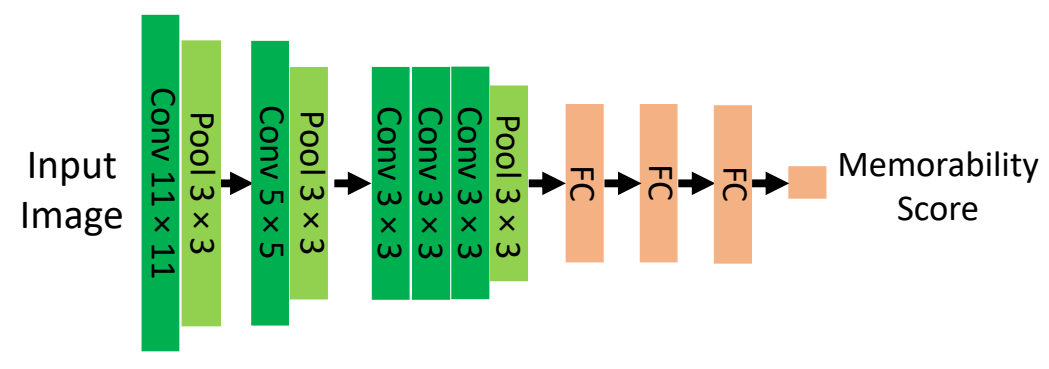

Figure 1. Architecture of MemNet

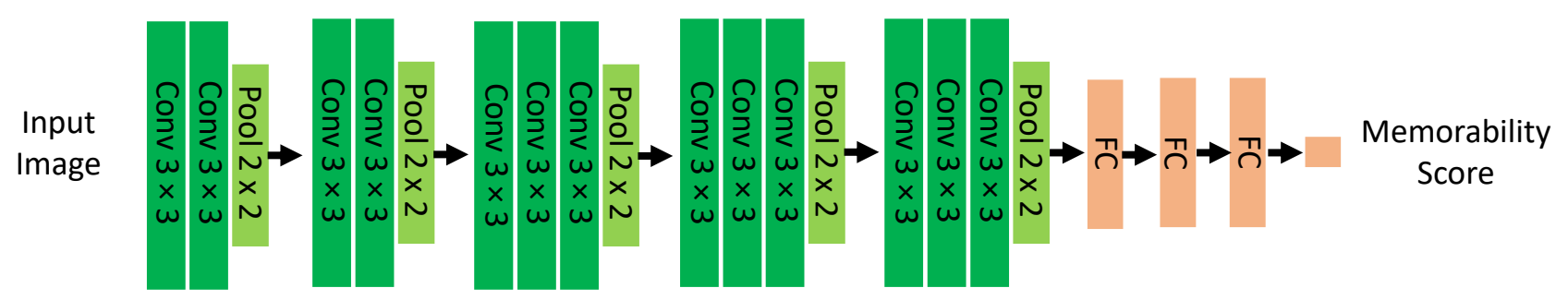

Figure 2. Architecture of MemVGG

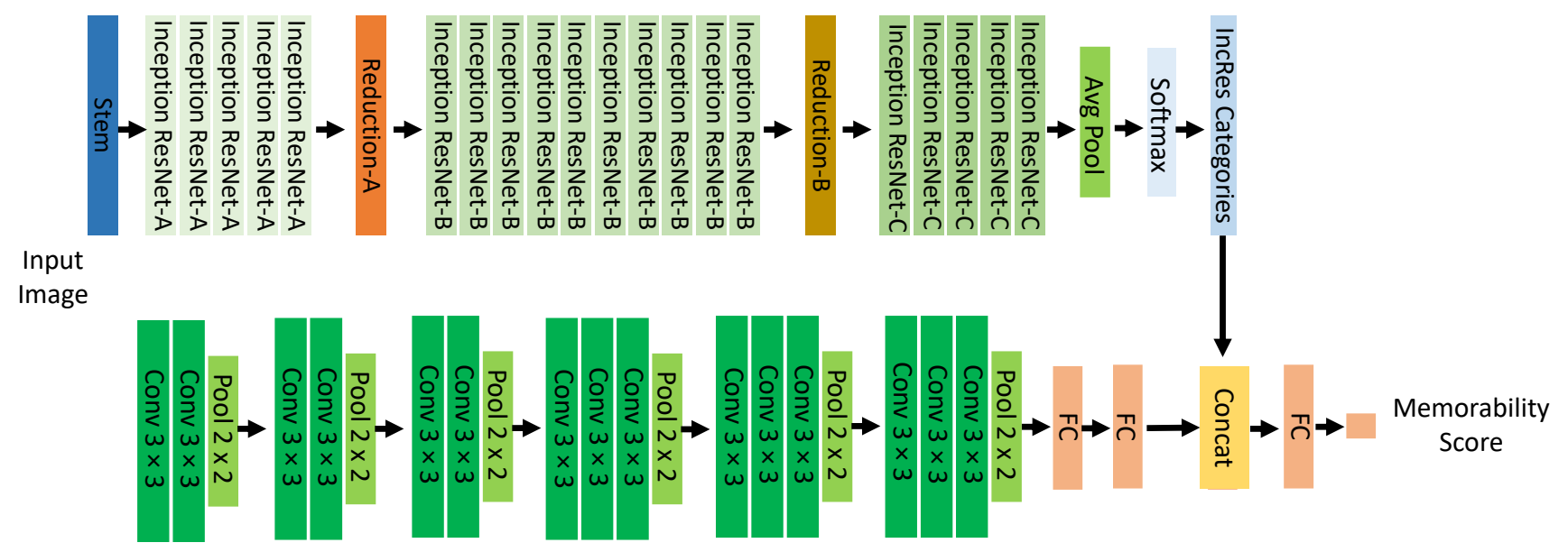

Figure 3. Architecture of IncResMem. The input image goes through two parallel streams and at last stage, the extracted features are combined to predict the memorability.

After training these models on LaMem and acquiring valid models for predicting image memorability, we investigated how well they can predict memorability of face images. We observed these models perform poorly in estimating face memorability. The results of this experiment are presented in Table 1. The distribution of the predicted face memorability scores of the mentioned three models is shown in Figure 4. As depicted, these models clearly fail to predict memorability scores for face images. As a result, in the next section we propose and train new models for face memorability prediction.

\section{MemFaceNet: Memorability models for faces}

As we demonstrated in previous section, the models that were trained on the LaMem dataset did not show acceptable performance on predicting face memorability scores. We propose ten new models for predicting face memorability in two groups. The first group consists of the three computational models that are pre-trained on LaMem dataset. We introduced these 


\begin{tabular}{c|c|c} 
Model & Hit Rate Score & True Hit Rate Score \\
\hline \hline MemNet & -0.013 & -0.033 \\
\hline MemVGG & 0.028 & 0.114 \\
\hline InceptionResMem & -0.040 & 0.013
\end{tabular}

Table 1. Prediction results obtained by the three proposed Memorability models on 10k US Faces Database

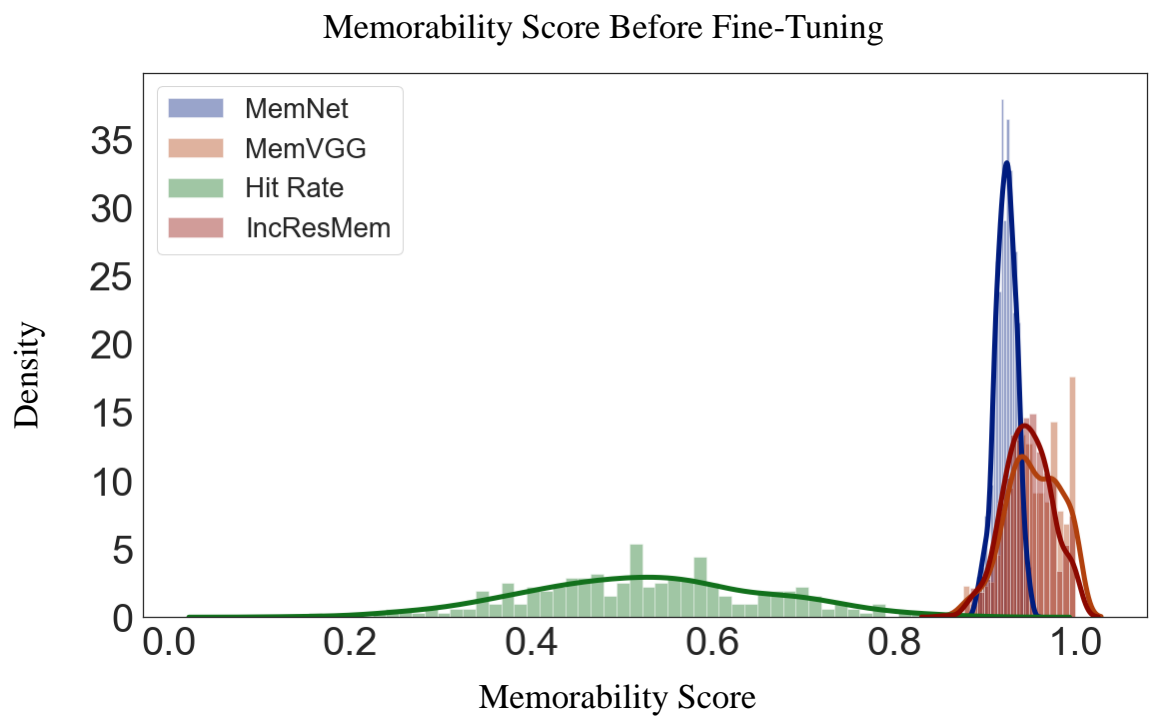

Figure 4. The distribution of ground truth face memorability scores and predictions of MemNet, MemVGG and IncResMem. The mean of the predictions are very high and close to one. The possible reason for this is that these models are trained on LaMem dataset where the images containing people are more memorable compared to images of objects and scenes. 
models (MemNet, MemVGG, and IncResMem) in the previous section. We fine-tuned these models on the 10K US faces dataset for predicting the memorability of faces. The second group includes seven models that are pretrained on VGGFace 2 dataset $^{31}$ and we fine-tuned them on 10k US faces dataset to estimate face memorability scores. Since the memorability dataset for face images is relatively small, the starting point of training process is crucially important and we thought that using pre-trained face models will help us in estimating the face memorability. This seven models include VGG16, ResNet50, SENET50 and all two by two and three by three combinations of features from these three models. We trained all these proposed models both with memorability scores computed by hit rates and the memorability scores corrected by false alarms.

\author{
Hit Rate $=\frac{\text { Number of Hits }}{\text { Number of Hits }+ \text { Number of Misses }}$ \\ Corrected Hit Rate = Hit Rate - False Alarm Rate \\ where : False Alarm Rate $=\frac{\text { Number of False Alarms }}{\text { Number of False Alarms }+ \text { Number of Correct Re jections }}$
}

Consistent with Khosla et al. ${ }^{16}$, we observed when the corrected hit rate scores are used, all models outperform the case when hit rate scores are used. That is because false alarm rate introduces noise to memorability scores, therefore, the models perform better when we reduce the noise by correcting for false alarms. Throughout this paper, we refer to the corrected hit rates as true hit rates and the uncorrected ones as hit rates. Moreover, the proposed models of both groups outperformed (see Table 2 and Table 3) the classic MemNet (see Table 1), which is used by Khosla et al. ${ }^{16}$ for predicting face memorability. We conclude that pre-trained face models had better representations of faces that were useful in predicting the face memorability scores and that is the reason why the models that were based on the pre-trained face models(group 2) performed relatively better than memorability networks (group 1) when fine-tuned to predict face memorability. The rank correlation performance of all models are presented in Table 2 and Table 3. We should add that Human consistency for the 10k US Faces Database is equal to 0.68 and 0.69 respectively when hit rate and corrected hit rate scores are used. SeNet and SenVGG resulted higher correlation rank compared to the other models. These models employ squeeze and excitation blocks which are beneficial in improving the representational power of the network. These squeeze and excitation blocks are used before summation and also with the identity branch. Simple schema of how these blocks are used has been shown in Figure 7.

We plotted the prediction distribution for all the models (see Figure 5 and Figure 6) and following Khosla et al. (2015) used linear transformation to make the mean and variance of the prediction distribution equal to the mean and variance of the train data. It is trivial that this linear transformation would not affect the rank correlation. The mean and variance of the hit rate for the training data is $(0.515,0.016)$ and the mean and variance of the corrected hit rate is equal to $(0.370,0.022)$ for the training data.

\begin{tabular}{c|c|c} 
Model & Hit Rate Score & True Hit Rate Score \\
\hline \hline FaceMemNet & $\mathbf{0 . 4 2 4}$ & $\mathbf{0 . 5 4 3}$ \\
\hline FaceMemVGG & 0.365 & 0.518 \\
\hline FaceInceptionResMem & 0.371 & 0.525
\end{tabular}

Table 2. Memorability scores of the models pre-trained on LaMem dataset and fine-tuned on 10k US face database. Fine-tuned MemNet model (FaceMemNet) performed better than FaceMemVGG and FaceInceptionResMem in predicting face memorability scores.

The important thing about the distribution plots that should be noticed is that, if the ground truth and prediction distribution align well together, it does not mean the model has performed well. It is necessary but it is not sufficient. The rank correlation should be considered for evaluation of the model. 


\begin{tabular}{c|c|c} 
Model & Hit Rate Score & True Hit Rate Score \\
\hline \hline VGG16 & 0.445 & 0.579 \\
\hline ResNet50 & 0.433 & 0.607 \\
\hline SeNet50 & 0.448 & 0.601 \\
\hline ResVGG & 0.423 & 0.626 \\
\hline SenRes & 0.452 & 0.631 \\
\hline SenVGG & $\mathbf{0 . 4 6 8}$ & 0.605 \\
\hline SenResVGG & 0.445 & $\mathbf{0 . 6 3 4}$
\end{tabular}

Table 3. Memorability scores of the models pre-trained on face recognition (on VGGfaces database) and fine-tuned on 10K US face database. The results are close to each other, however we can observe that SenRes and SenResVGG show better performance in comparison with other models. Note all these computational models, produce larger Spearman's rank correlation score when true hit rate scores (corrected scores) are used.

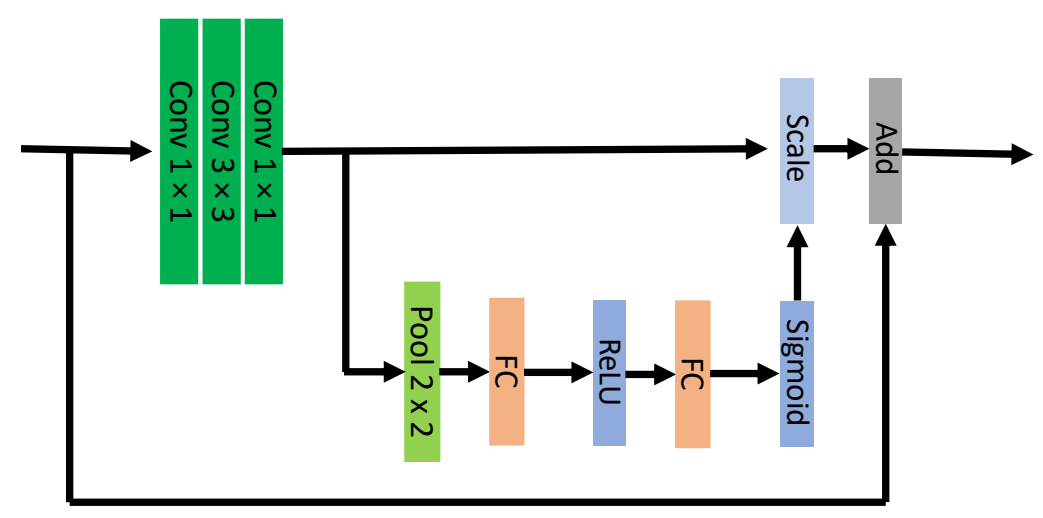

Figure 7. Simple schema of squeeze and excitation block used with identity branch of SENet

\section{Memorability of oval-shaped and square-shaped faces}

The 10K US face dataset contains oval-shaped faces with white background. However, it is time and resource consuming to convert all the face images with the format of this database, whenever we want to predict the face memorability score. Therefore, we decided to test our models on the same set of oval-shaped and square-shaped faces. We utilized StyleGan2 ${ }^{37}$ pretrained on FFHQ datase ${ }^{38}$, to generate $8 \mathrm{k}$ high-quality and realistic face images. The generated faces from the StyleGan2 have $1024 \times 1024$ resolution in three channels. We changed their size in the pre-processing step, and then calculated their memorability scores with all our models. In order to ovalize these images, we leveraged MTCNN $^{39}$ to detect the coordinates of the faces in the image and then masked an oval on it to make them in format of the 10k US face database (See Figure 8). Table 4 shows the Spearman's rank correlation of predicted memorability scores for the oval-shaped and square-shaped images. We observe that except for SenVGG, other models that were pre-trained on VGGFace2 and then fine-tuned with the 10K US face database, result in higher correlation scores compared to the models which are first fune-tuned on LaMem, then fine-tuned on the 10K US face database. 


\section{Ground Truth}

Predictions

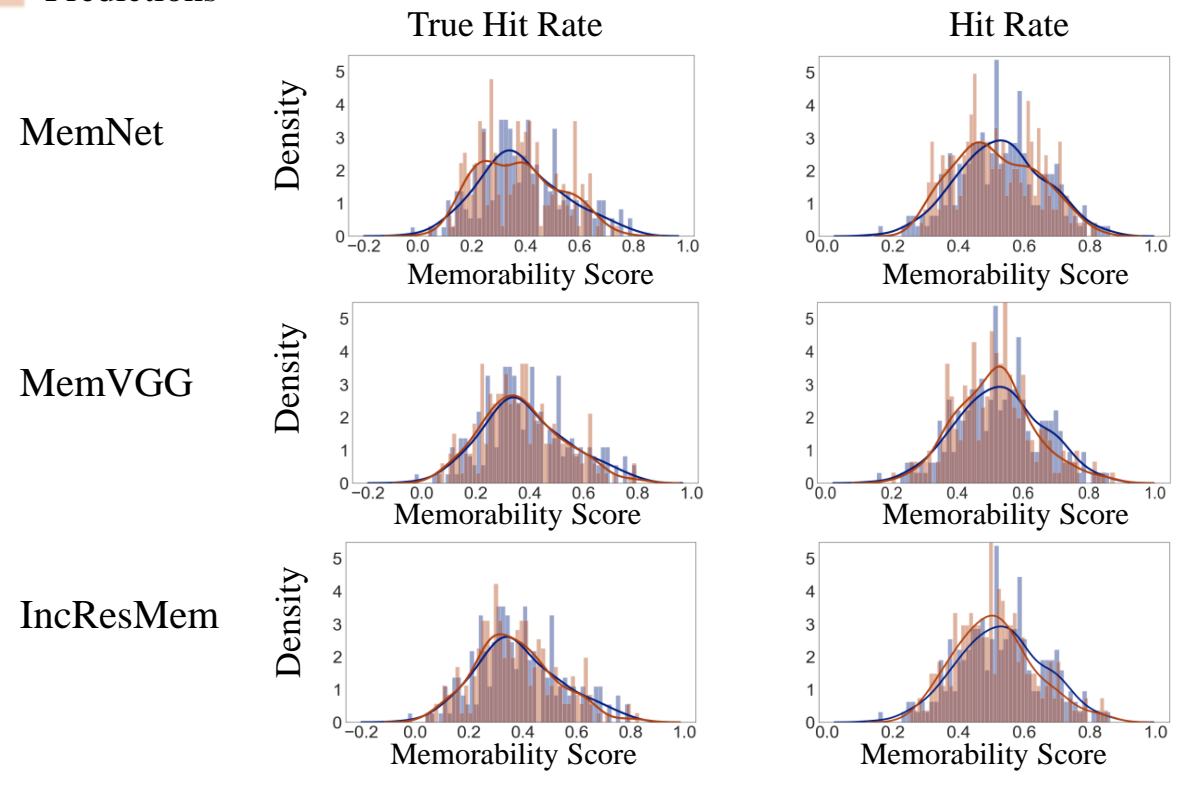

Figure 5. Prediction distributions of the models pre-trained on LaMem and fine-tuned on 10k US Face Database in comparison with ground truth scores on both hit rate (uncorrected scores) and true hit rate scores (false alarm corrected scores)

\begin{tabular}{l|c} 
Assessor & Spearman's correlation \\
\hline \hline FaceMemNet & 0.6976 \\
\hline FaceMemVGG & 0.2476 \\
\hline FaceInceptionResMet & 0.3687 \\
\hline ResNet50 & 0.4933 \\
\hline SENet50 & 0.6217 \\
\hline VGG16 & 0.6562 \\
\hline ResVGG & 0.6793 \\
\hline SenRes & 0.6668 \\
\hline SenVGG & 0.3520 \\
\hline SenResVGG & 0.6609
\end{tabular}

Table 4. Rank correlations of predicted memorability scores of square-shaped and oval-shaped face images. High correlation score suggests the memorability models perform well on square-shaped faces too.

\section{Discussion}

Focusing on face images, we showed the existing models used in predicting image memorability fail to predict face memorability scores. We specifically first evaluated $\mathrm{MemNet}^{11}$, which is trained on LaMem ${ }^{11}$ dataset. Moreover, we leveraged two other convolutional neural network model architectures (VGG16 ${ }^{28}$ and InceptionResNet ${ }^{35}$ ) and trained them on LaMem dataset for prediction of image memorability. These three models showed great performance in predicting memorability of object, scene and animal images on LaMem Dataset, albeit as we expected, they failed to predict the face memorability scores in 10K US face dataset. The main reason is that these models are trained on LaMem which is a large memorability dataset containing images of objects and scenes. We employed 10K US Face Database ${ }^{3}$ to fine-tune these three models and observed the rank correlation score significantly increased. In addition to the three mentioned models, we proposed seven new models for estimating face memorability scores. These seven models are based on the-state-of-the-art pre-trained face recognition models ${ }^{28-31}$, which we then fine-tuned them to predict the face memorability. We hypothesized these face recognition models extract more efficient facial features and will be a better back-bone to fine-tune for predicting face memorability. Further, these models have a 


\section{Ground Truth}

Predictions

True Hit Rate

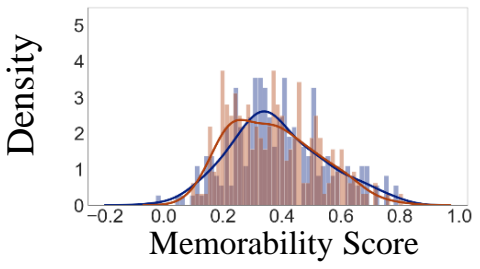

ResNet50

SeNet50

ResVGG
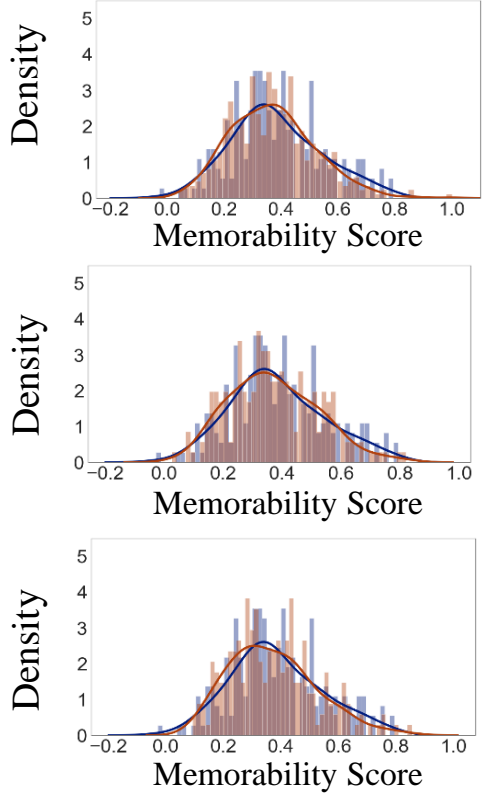

SenVGG

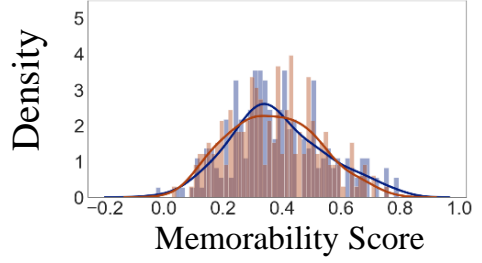

SenRes

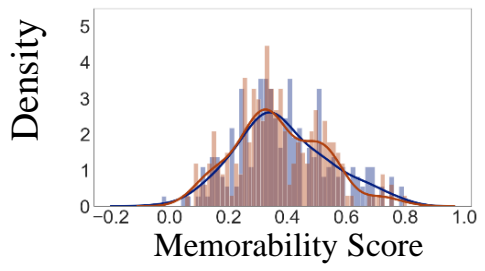

SenResVGG

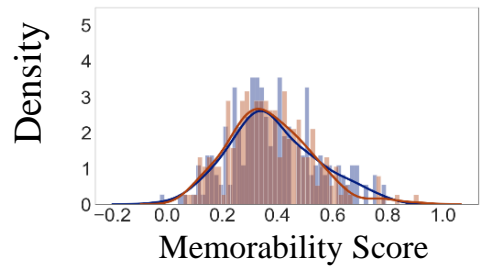

Hit Rate
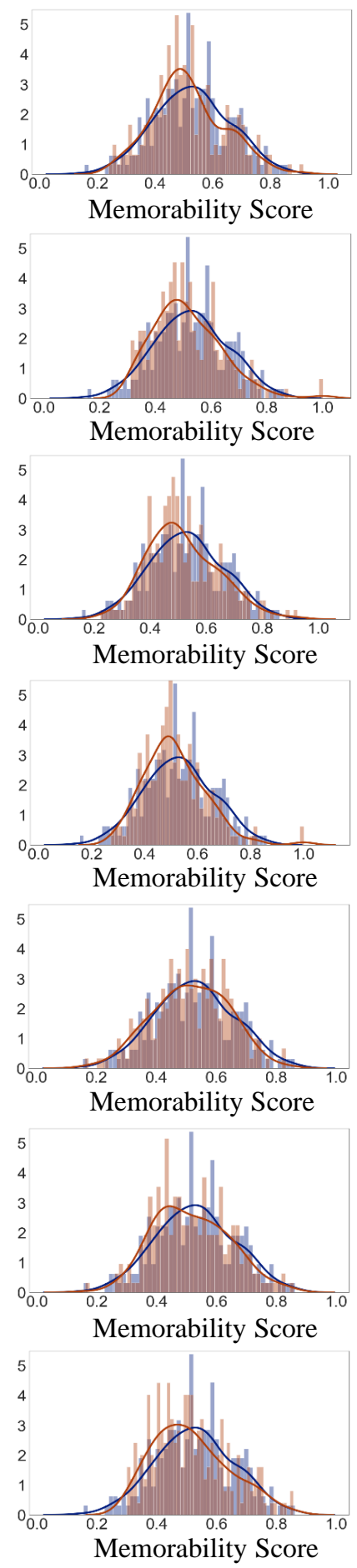

Figure 6. Prediction distributions of the models pre-trained on face recognition task (on VGGFace Database) and fine-tuned on 10k US Face Database in comparison with ground truth scores on both hit rate and true hit rate scores 


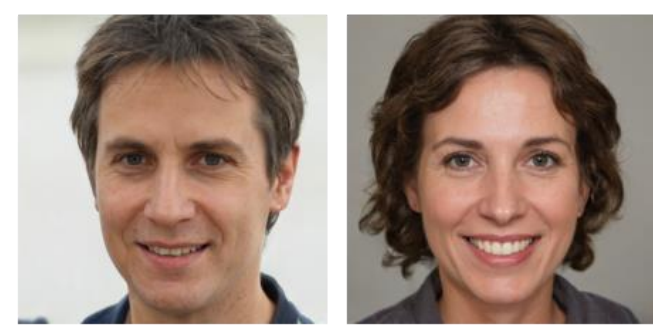

Sample generated face images by StyleGan2

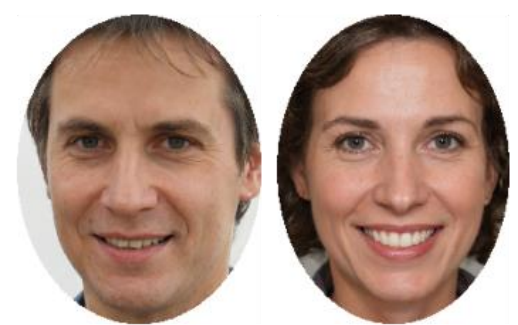

Sample images after ovalizing step

Figure 8. Ovalizing step to make the shape of the synthesized images similar to the dataset that the assessor is trained on

simpler pre-processing step compared to the previous models like MemNet. The backbone of these seven models are VGG16 ${ }^{28}$, SENET $50^{30}$ and ResNet50 $0^{29}$, which all three of them have shown great performance in numerous machine learning tasks like face recognition. We observed models pre-trained on faces, show a better performance in predicting face memorability. This confirms our hypothesis that these models extract features from faces that are more useful for predicting face memorability.

The proposed models in this work pave the way for predicting memorability of face images in new datasets. Acquiring memorability scores for images requires running large-scale visual memory experiments and crowd sourcing participants usually on online platforms like Amazon Mechanical Turk. These experiments are time-consuming and costly. Having these models will remove this barrier and provides a great opportunity to run future experiments on face memorability.

\section{Methods}

\section{Deep convolutional neural networks for face memorability prediction}

It is common knowledge that deep convolutional neural networks have great power in different machine learning tasks like regression and classification. In this work, we trained two models (MemVGG, IncResMem) on LaMem with addition of original MemNet model for predicting image memorability then fine-tuned them on 10k US Adult Faces Database. The backbone of MemNet, MemVGG and IncResMem are AlexNet, VGG16 and (InceptionResNetv2 and VGG16), respectively. The backbone models of MemNet and MemVGG are preetrained on both object categories(ImageNet) and scene categories(Places). For the InceptionResMem model, we used pre-trained InceptionResnet model which was pre-trained on Imagenet with addition to a hybrid pre-trained VGG, so we took advantage of both Imagenet and Places classes for predicting memorability scores. The reason behind this choice is that the LaMem dataset is very diverse; It contains objects, scenes and also combination of objects and scenes. Consequently, to ensure that the model extracts good representation of these images, we chose hybrid models for the backbone of our models. In MemNet, Alexnet features are used, followed by three fully connected layers. The input images were first resized $(256,256)$ pixels with a bi-linear transformation then images were cropped to a $(224,224)$ square shape from the center of the image. All the input images were normalized to the range between 0 and 1 . The batch size is 64 in all of the models and Euclidean distance is used as the loss function. The MemVGG model is very similar to MemNet, except the fact that VGG16 is used as the backbone of the model and VGG16 features are used instead of the AlexNet features. In this model, we didn't need to normalize the input images between 0 and 1 . The last model, which we trained on LaMem dataset is IncResMem. Its architecture is shown in Figure 3. InceptionResNetv2 achieves great performance in image classification task on ImageNet and also can be trained much faster than ResNet models. We combined InceptionResNet categories and VGG16 features, so the layer before the output includes 5096 nodes. The output predicts the memorability of images. There are two separated parallel branch in this network. The size of the input for the VGG16 branch is $(224,224)$ and for the InceptionResnetv2 branch is $(299,299)$.

After training these models on LaMem, we tested them for predicting the memorability of the face images. We checked them on 10k US Adult Face Database and as we expected, these models failed to predict memorability of faces. As a result, using transfer learning principles, we fine-tuned these three models on US 10k Adult Face Database.

In order to train the models, we split the 10k US Face Database images into train, validation and test split. We used 80 percent of the data as the training samples and used 10 percent of the data for each of the test and validation splits. Again, Euclidean distance was used as the loss function and we set the batch size to 64. Moreover, we leveraged Adam optimizer to train our models. Due to large false alarm rate in human face images, we trained our models both with raw memorability scores (computed by hit rate) and corrected memorability scores (considering false alarm rate). We also tried some simple 
augmentations on the dataset and found, the score of the models will slightly increase if we use simple augmentation like random horizontal flipping $(p=0.5)$.

As we mentioned in the Result section, following Khosla et al. (2015), we used linear transformation to set the mean and variance of the predicted memorability samples equal to the train data.

$$
\begin{array}{r}
\text { mean }_{\left(\text {pred }_{i}^{\text {new }}\right)}=\text { mean }\left[\left(\text { pred }_{i}^{\text {old }}-\text { mean }_{\text {predicted }}\right) \times \frac{s t d_{\text {train }}}{s t d_{\text {predicted }}}+\text { mean }_{\text {train }}\right] \\
=\text { mean }\left[\left(\text { pred }_{i}^{\text {old }}-\text { mean }_{\text {predicted }}\right) \times \frac{s t d_{\text {train }}}{s t d_{\text {predicted }}}\right]+\text { mean }_{\left(\text {mean }_{\text {train }}\right)} \\
=\text { mean }\left[\left(\text { pred }_{i}^{\text {old }}-\text { mean }_{\text {predicted }}\right)\right] \times \frac{s t d_{\text {train }}}{s t d_{\text {predicted }}}+\text { mean }_{\text {train }} \\
=\left(\text { mean }_{\text {predicted }}-\text { mean }_{\text {predicted }}\right) \times \frac{s_{\text {train }}}{\text { std }_{\text {predicted }}}+\text { mean }_{\text {train }}=\text { mean }_{\text {train }}
\end{array}
$$

Equivalently, we can show this similarly for the variances.

Deep convolutional neural networks are capable of extracting the most generic features when they are trained on huge datasets. While we are dealing with face images within a considerable small dataset, we decided to use deep models that are pre-trained on a large faces dataset. Therefore, we utilized SeNet50, ResNet50 and VGG16 that are pre-trained on VGGFace2 dataset $^{31}$. This dataset contains more than 3.3 million images within 9131 subjects. Images in this dataset have large variations in pose, age, illumination, ethnicity and profession.

Then, we introduce seven new memorability predicting models by leveraging SeNet50, ResNet50 and VGG16. These models can be divided into three groups. In the first group, we fine-tuned these three models with 10k US Faces Database to predict face memorability. Features of the last layer from each of the three mentioned models were combined together two by two and formed the second group. Then we fine-tuned them to predict face memorability scores. ResVGG was produced by combining the features from VGG16 network and the ResNet network, SenVGG was created from combining the SeNet50 features and the VGG16 features, and a combination of the SeNet50 and ResNet50 features was used to build the SenRes model. Finally, we combined features from all these three models and proposed the SenResVGG network. We trained all models with 0.5 chance of horizontal mirroring of the images as an augmentation. We observed that this augmentation helps overcoming over-fitting problem and also increases the rank correlation score.

\section{References}

1. Hunt, E. B., Davidson, J. \& Lansman, M. Individual differences in long-term memory access. Mem. $\mathcal{G}$ Cogn. 9, 599-608 (1981).

2. Brady, T. F., Konkle, T., Alvarez, G. A. \& Oliva, A. Visual long-term memory has a massive storage capacity for object details. Proc. Natl. Acad. Sci. 105, 14325-14329 (2008).

3. Bainbridge, W. A., Isola, P. \& Oliva, A. The intrinsic memorability of face photographs. J. experimental psychology. Gen. 142 4, 1323-34 (2013).

4. Isola, P., Xiao, J., Torralba, A. \& Oliva, A. What makes an image memorable? In CVPR 2011, 145-152 (IEEE, 2011).

5. Isola, P., Xiao, J., Parikh, D., Torralba, A. \& Oliva, A. What makes a photograph memorable? Pattern Analysis Mach. Intell. IEEE Transactions on 36, 1469-1482 (2014).

6. Borkin, M. A. et al. What makes a visualization memorable? IEEE transactions on visualization computer graphics 19, 2306-2315 (2013).

7. Isola, P., Parikh, D., Torralba, A. \& Oliva, A. Understanding the intrinsic memorability of images. In Shawe-Taylor, J., Zemel, R., Bartlett, P., Pereira, F. \& Weinberger, K. Q. (eds.) Advances in Neural Information Processing Systems, vol. 24 (Curran Associates, Inc., 2011).

8. Khosla, A., Xiao, J., Isola, P., Torralba, A. \& Oliva, A. Image memorability and visual inception. In SIGGRAPH Asia 2012 technical briefs, 1-4 (2012).

9. Goetschalckx, L. \& Wagemans, J. Memcat: A new category-based image set to study image memorability. DOI: 10.13140/RG.2.2.19105.15202 (2019).

10. Almog, G., Naeini, S. A., Hu, Y., Duerden, E. \& Mohsenzadeh, Y. Memoir dataset: Quantifying image memorability in adolescents. . 
11. Khosla, A., Raju, A. S., Torralba, A. \& Oliva, A. Understanding and predicting image memorability at a large scale. In 2015 IEEE International Conference on Computer Vision (ICCV), 2390-2398, DOI: 10.1109/ICCV.2015.275 (2015).

12. Newell, F. N., Chiroro, P. \& Valentine, T. Recognizing unfamiliar faces: The effects of distinctiveness and view. The $Q$. $J$. Exp. Psychol. Sect. A 52, 509-534 (1999).

13. Light, L. L., Kayra-Stuart, F. \& Hollander, S. Recognition memory for typical and unusual faces. J. Exp. Psychol. Hum. Learn. Mem. 5, 212 (1979).

14. Light, L. L., Hollander, S. \& Kayra-Stuart, F. Why attractive people are harder to remember. Pers. Soc. Psychol. Bull. 7, 269-276 (1981).

15. Bartlett, J. C., Hurry, S. \& Thorley, W. Typicality and familiarity of faces. Mem. $\mathcal{G}$ Cogn. 12, 219-228 (1984).

16. Khosla, A., Bainbridge, W. A., Torralba, A. \& Oliva, A. Modifying the memorability of face photographs. In Proceedings of the IEEE International Conference on Computer Vision, 3200-3207 (2013).

17. Dalal, N. \& Triggs, B. Histograms of oriented gradients for human detection. In 2005 IEEE computer society conference on computer vision and pattern recognition (CVPR'05), vol. 1, 886-893 (Ieee, 2005).

18. Lindeberg, T. Scale invariant feature transform. (2012).

19. LeCun, Y., Bengio, Y. et al. Convolutional networks for images, speech, and time series. The handbook brain theory neural networks 3361, 1995 (1995).

20. Krizhevsky, A., Sutskever, I. \& Hinton, G. E. Imagenet classification with deep convolutional neural networks. Adv. neural information processing systems 25, 1097-1105 (2012).

21. Zhou, B., Lapedriza, A., Xiao, J., Torralba, A. \& Oliva, A. Learning deep features for scene recognition using places database. (2014).

22. Fajtl, J., Argyriou, V., Monekosso, D. \& Remagnino, P. Amnet: Memorability estimation with attention. arxiv 2018. arXiv preprint arXiv:1804.03115.

23. Needell, C. D. \& Bainbridge, W. A. Embracing new techniques in deep learning for estimating image memorability. arXiv preprint arXiv:2105.10598 (2021).

24. Lu, J., Xu, M., Yang, R. \& Wang, Z. Understanding and predicting the memorability of outdoor natural scenes. IEEE Transactions on Image Process. 29, 4927-4941 (2020).

25. Bylinskii, Z., Isola, P., Bainbridge, C., Torralba, A. \& Oliva, A. Intrinsic and extrinsic effects on image memorability. Vis. research 116, 165-178 (2015).

26. Bainbridge, W. A. The memorability of people: Intrinsic memorability across transformations of a person's face. J. Exp. Psychol. Learn. Mem. Cogn. 43, 706 (2017).

27. Squalli-Houssaini, H., Duong, N. Q., Gwenaëlle, M. \& Demarty, C.-H. Deep learning for predicting image memorability. In 2018 IEEE International Conference on Acoustics, Speech and Signal Processing (ICASSP), 2371-2375 (IEEE, 2018).

28. Simonyan, K. \& Zisserman, A. Very deep convolutional networks for large-scale image recognition. arXiv preprint arXiv:1409.1556 (2014).

29. He, K., Zhang, X., Ren, S. \& Sun, J. Deep residual learning for image recognition. In Proceedings of the IEEE conference on computer vision and pattern recognition, 770-778 (2016).

30. Hu, J., Shen, L. \& Sun, G. Squeeze-and-excitation networks. In Proceedings of the IEEE conference on computer vision and pattern recognition, 7132-7141 (2018).

31. Cao, Q., Shen, L., Xie, W., Parkhi, O. M. \& Zisserman, A. Vggface2: A dataset for recognising faces across pose and age. In 2018 13th IEEE international conference on automatic face $\mathcal{F}$ gesture recognition (FG 2018), 67-74 (IEEE, 2018).

32. Paszke, A. et al. Pytorch: An imperative style, high-performance deep learning library. In Wallach, H. et al. (eds.) Advances in Neural Information Processing Systems 32, 8024-8035 (Curran Associates, Inc., 2019).

33. Deng, J. et al. Imagenet: A large-scale hierarchical image database. In 2009 IEEE conference on computer vision and pattern recognition, 248-255 (Ieee, 2009).

34. Zhou, B., Lapedriza, A., Khosla, A., Oliva, A. \& Torralba, A. Places: A 10 million image database for scene recognition. IEEE transactions on pattern analysis machine intelligence 40, 1452-1464 (2017).

35. Szegedy, C., Ioffe, S., Vanhoucke, V. \& Alemi, A. A. Inception-v4, inception-resnet and the impact of residual connections on learning. In Thirty-first AAAI conference on artificial intelligence (2017). 
36. Chollet, F. et al. Keras. https://keras.io (2015).

37. Karras, T. et al. Analyzing and improving the image quality of stylegan. In Proceedings of the IEEE/CVF Conference on Computer Vision and Pattern Recognition, 8110-8119 (2020).

38. Karras, T., Laine, S. \& Aila, T. A style-based generator architecture for generative adversarial networks. In Proceedings of the IEEE/CVF Conference on Computer Vision and Pattern Recognition, 4401-4410 (2019).

39. Zhang, K., Zhang, Z., Li, Z. \& Qiao, Y. Joint face detection and alignment using multitask cascaded convolutional networks. IEEE Signal Process. Lett. 23, 1499-1503 (2016).

\section{Acknowledgements}

This study was supported by the Canada First Research Excellence Fund (CFREF) through Western's BrainsCAN Initiative. The Computational modelling was conducted on Compute Canada resources. M.Y. was supported by a Vector Institute Masters Scholarship in Artificial Intelligence.

\section{Author contributions statement}

M.Y. and Y.M. conceived the study, M.Y. conducted the modelings and experiments, M.Y., and Y.M. analysed the results and wrote the manuscript.

\section{Additional information}

Competing interests The authors declare no competing interests. 